\title{
EFFECT OF ELECTRICAL CABLE ON MOVEMENT-RANGE OF AGNETIC ACTUATOR SYSTEM CAPABLE OF INSPECTION IN A PIPE
}

\author{
K. Sasaki ${ }^{1}$, T. Sato ${ }^{1}$ and H. Yaguchi ${ }^{1}$ \\ ${ }^{1}$ Faculty of Mechanical Engineering, Tohoku Gakuin University, \\ 1-13-1 Chuo, Tagajo, Japan \\ *Email: yaguchi@mail.tohoku-gakuin.ac.jp \\ Phone: +81 223687104; Fax: +81 223687070
}

\begin{abstract}
This paper proposes a magnetic actuator system of a new type capable of inspection in a complex pipe by means of a new motion principle. Using plural vibration components, the magnetic actuator is able to move in the complex pipe. A magnetic actuator of the reversible type, due to opening and closing of a compound material by using elongation and contraction of four shape-memory-alloy (SMA) coils, was fabricated. In this paper, the influence of the electrical cable length on the moving characteristics of the magnetic actuator is discussed. Twenty electrical cables of $0.12 \mathrm{~mm}$ diameter were used in this paper. The experimental result shows that the prototype actuator was able to climb at $15.3 \mathrm{~mm} / \mathrm{s}$ in the straight pipe when pulling a load mass of $50 \mathrm{~g}$. In addition, the result demonstrated that the magnetic actuator system was able to inspect the complex pipe over a distance of $10 \mathrm{~m}$. This actuator system can inspect a complex pipe with an inside diameter from $40 \mathrm{~mm}$ to $50 \mathrm{~mm}$. In addition, the actuator system was able to inspect the pipe with a simple apparatus of three amplifiers and one signal generator.
\end{abstract}

Keywords: Magnetic actuator system; pipe inside mover; frictional force; inspection.

\section{INTRODUCTION}

An actuator capable of movement in a complex pipe is required to quickly find damage in a chemical plant, heat exchanger, nuclear reactor or other such installations. On the other hand, pipes with an inner diameter of $45 \mathrm{~mm}$ used in water service and gas pipelines are buried underground and are connected like a mesh or network all over the world, so pipelines are a social base that is indispensable to life. Maintenance of pipelines is essential to maintain the soundness of these lifelines. A robot capable of visual inspection inside complex pipes is required. A number of studies have investigated the mechanisms for control of robots by means of an electrical cable to provide locomotion in a pipe, using devices such as piezoelectric elements [1-3], shape memory alloys [4, 5], and electromagnetic motors [6-11]. Several kinds of inspection robot have been demonstrated. However, a freely movable robot in a small inner diameter pipe of $45 \mathrm{~mm}$ with many curved parts and step parts has not been much studied except by Yaguchi et al. [12]. Using the main component of the robot and a very simple device, No system capable of inspection of complex pipes using just the main component of the robot and a very simple device has yet been reported. The authors [13, 14] previously proposed a novel magnetic actuator that provides propulsion by a new motion principle. In particular, the actuator shown by Yaguchi et al. [15] can move in a complex pipe with a T-junction and steps. However, the motion properties of the 
actuator have not yet been examined sufficiently. Accordingly, the influence of the electrical cable length on the moving properties of the actuator system has not been determined. In general, as the length of the electrical cable increases, the frictional force between the wall of the inner pipe and the electrical cable increases.

The purpose of this present study is to completely elucidate the influence of the electrical cable length on the moving characteristics of the magnetic actuator system presented in the previous paper [12]. The effect of the electrical cable on the moving properties of the magnetic actuator system is discussed experimentally. In addition, we demonstrate that the actuator system was able to inspect the complex pipe by using a simple apparatus of only three amplifiers and one signal generator. Experimental results show that the presented actuator system can inspect a pipe $45 \mathrm{~mm}$ in diameter over a distance of approximately $10 \mathrm{~m}$.

\section{MAGNETIC ACTUATOR SYSTEM CAPABLE OF FREELY INSPECTING THE COMPLEX PIPE}

Figure 1 shows all the vibration components of the magnetic actuator system capable of movement in the complex pipe. Vibration component 1 was attached on an acrylic frame. Vibration components 2 and 3 were attached at a right angle to vibration component 1 . In a permanent magnet and spring of all the vibration components, it is the same properties. The size and basic structure of the magnetic actuator system is the same as shown in the previous study [15]. The permanent magnet was attached to the spring. The mass-spring system, composed of the electromagnet and the translational spring, was adhered to the acrylic frame. The translational spring is a stainless steel type with an outer diameter of $9 \mathrm{~mm}$, and a free length of $14 \mathrm{~mm}$. The permanent magnet is cylindrical $\mathrm{NdFeB}$ and is magnetized in the axial direction. It is 9 $\mathrm{mm}$ in diameter and its height is $3 \mathrm{~mm}$. The surface magnetic flux density as measured using a tesla meter was $306 \mathrm{mT}$. All the permanent magnets were shielded by iron to prevent mutual interference when all the vibration components vibrated, as shown in Figure 1.

Table 1. Specifications of the spring and electromagnet.

\begin{tabular}{ll}
\hline \multicolumn{1}{c}{ Name } & Value \\
\hline Spring constant $(\mathrm{N} / \mathrm{m})$ & 2821 \\
Diameter of spring $(\mathrm{mm})$ & 9 \\
Length of spring $(\mathrm{mm})$ & 14 \\
Coil trun of electromagnet at vibration body 1 & 900 \\
Coil turn of electromagnet at vibration body 2 and 3 & 352 \\
Resistance of electromagnet at vibration body 1 $(\Omega)$ & 5,1 \\
Resistance of electromagnet at vibration body 2 and 3 $(\Omega)$ & 1.7 \\
\hline
\end{tabular}

For vibration component 1 , the electromagnet consists of an iron core $2.2 \mathrm{~mm}$ in diameter and $25 \mathrm{~mm}$ in length with 900 turns of $0.12 \mathrm{~mm}$ diameter copper wire. For vibration components 2 and 3, the electromagnet consists of an iron core $3 \mathrm{~mm}$ in diameter and $10 \mathrm{~mm}$ in length with 352 turns of $0.12 \mathrm{~mm}$ diameter copper wire. The specifications of the spring and electromagnet are given in Table 1. The gap between the electromagnet and the permanent magnet in the static condition is $3 \mathrm{~mm}$. 

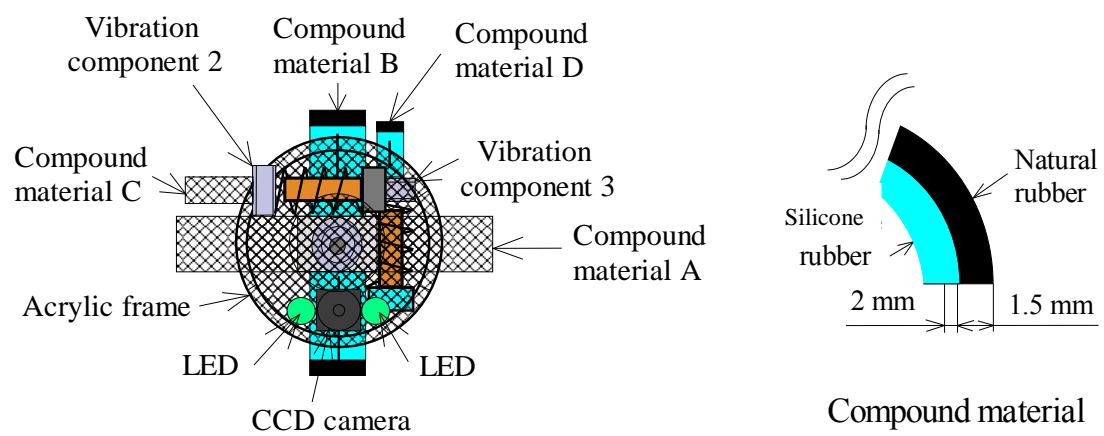

Plan

Compound material

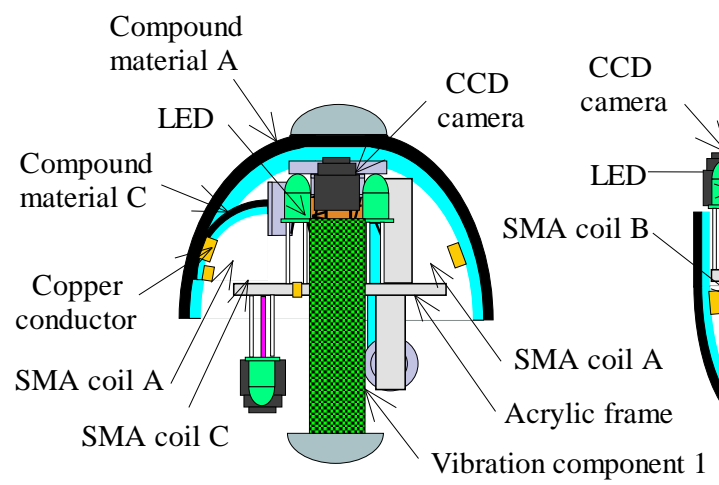

Acrylic cap

Front view

$\downarrow$ Vibration component 1
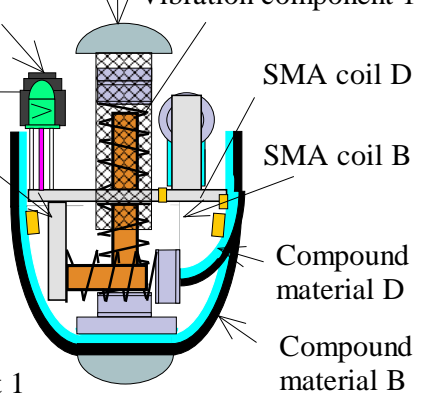

Side view

Figure 1. Structure of the magnetic actuator system capable of reversible motion.

The magnetic actuator is composed of a propulsion module as shown in Figure 1, the four shape-memory-alloy (SMA) coils, two layers of compound material A and $\mathrm{B}$, two layers of compound materials $\mathrm{C}$ and $\mathrm{D}$ for rotation and movement of the T-junction, eight copper conductors attached to the compound materials, and the acrylic frame. The SMA coil has a spring type shape with an outer diameter of $0.4 \mathrm{~mm}$ and length of $14 \mathrm{~mm}$ for compound materials A, B, C and D when it was completely contracted. The magnetic actuator undergoes linear and rotational movement based on the difference in frictional force between the forward and backward movement of the compound material [15]. Figure 1 also shows the structure of the magnetic actuator capable of inspecting the pipes. Two CCD cameras and four LED lights were attached to the acrylic frame to inspect inside the complex pipes. The actuator has a length of 56 $\mathrm{mm}$, a diameter of $38 \mathrm{~mm}$, and total mass of $27.2 \mathrm{~g}$. Figure 2 shows a photograph of the magnetic actuator system.

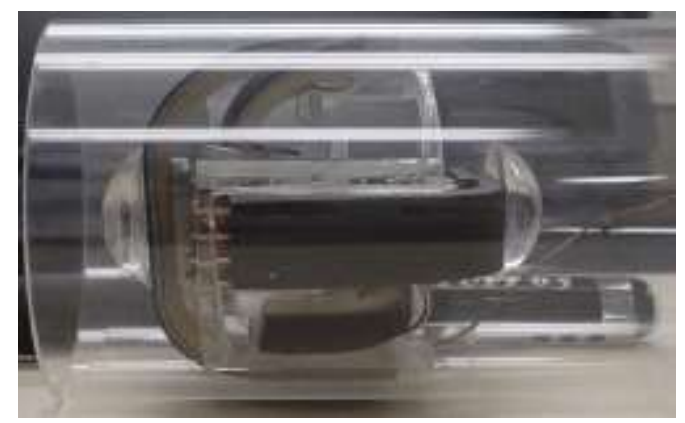

Figure 2. Photograph of the magnetic actuator system. 


\section{BASIC LOCOMOTION CHARACTERISTICS OF MAGNETIC ACTUATOR SYSTEM}

An experimental test was conducted using the apparatus shown in Figure 3. The magnetic actuator system is moved according to the vibration amplitude and elastic energy of the mass-spring system due to mechanical resonance energy. The resonance frequency of the magnetic actuator measured by using the experimental apparatus was $132 \mathrm{~Hz}$. In this measurement, fourteen electric cables (six cables to the three electromagnets and eight cables to the four SMA wires) were used. In this case, we measured without connecting the cables of the two CCD cameras and the four LED lights. The total cable length to the actuator system from each device is $1 \mathrm{~m}$. In the experiment, we confined the input electric current to approximately 0.2 A. Figure 4 shows the relationship between the load mass and the vertical upward speed for straight pipes with inner diameters of $40 \mathrm{~mm}, 45 \mathrm{~mm}, 48 \mathrm{~mm}$ and $50 \mathrm{~mm}$ when the input current into electromagnet 1 of vibration component 1 was $0.22 \mathrm{~A}$. This indicates that the magnetic actuator was able to climb at $15.3 \mathrm{~mm} / \mathrm{s}$ when pulling a $50 \mathrm{~g}$ load mass with an inner pipe diameter of $40 \mathrm{~mm}$ - a moderately high performance.

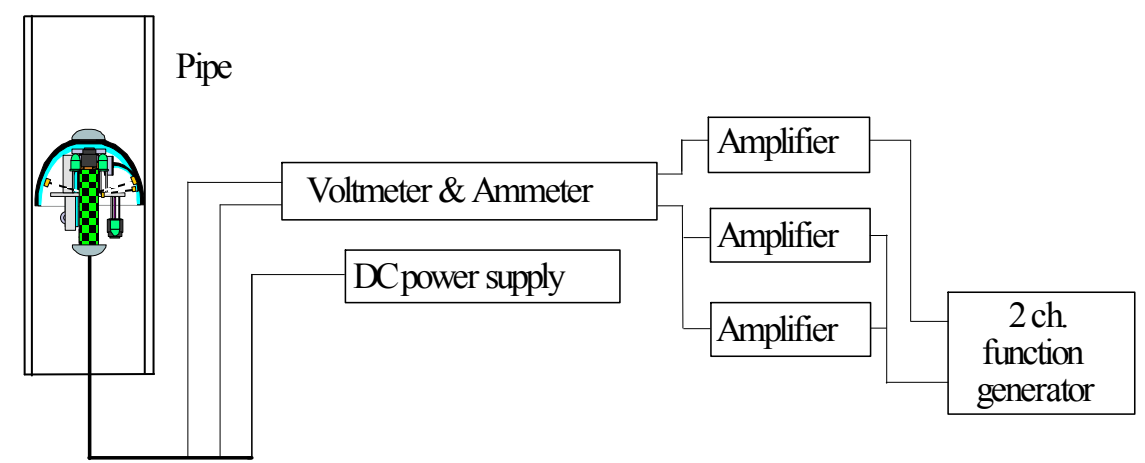

Figure 3. Measurement of speed for the moving actuator system.

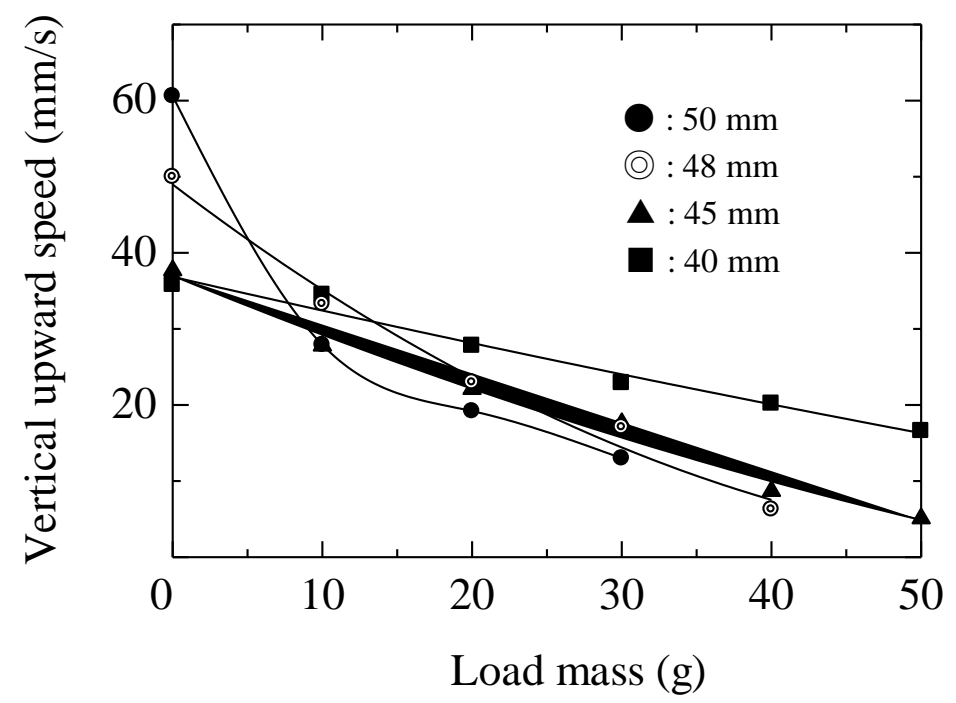

Figure 4. Relationship between load mass and vertical upward speed. 
Figure 5 shows the relationship between the tilt angle $\alpha$ of the straight pipe and the speed with regard to straight pipes with inner diameters of $40 \mathrm{~mm}, 45 \mathrm{~mm}, 48 \mathrm{~mm}$ and $50 \mathrm{~mm}$ when the actuator has no load mass. The tilt angle $\alpha$ was varied from $-90^{\circ}$ (straight down) to $90^{\circ}$ (straight up). In Figure 5, the maximum vertical upward speed was $36.4 \mathrm{~mm} / \mathrm{s}$ when the inner diameter of the pipe was $40 \mathrm{~mm}$. For the pipe with inner diameter of $50 \mathrm{~mm}$, the speed when moving straight down was about 2.1 times that when moving straight up.

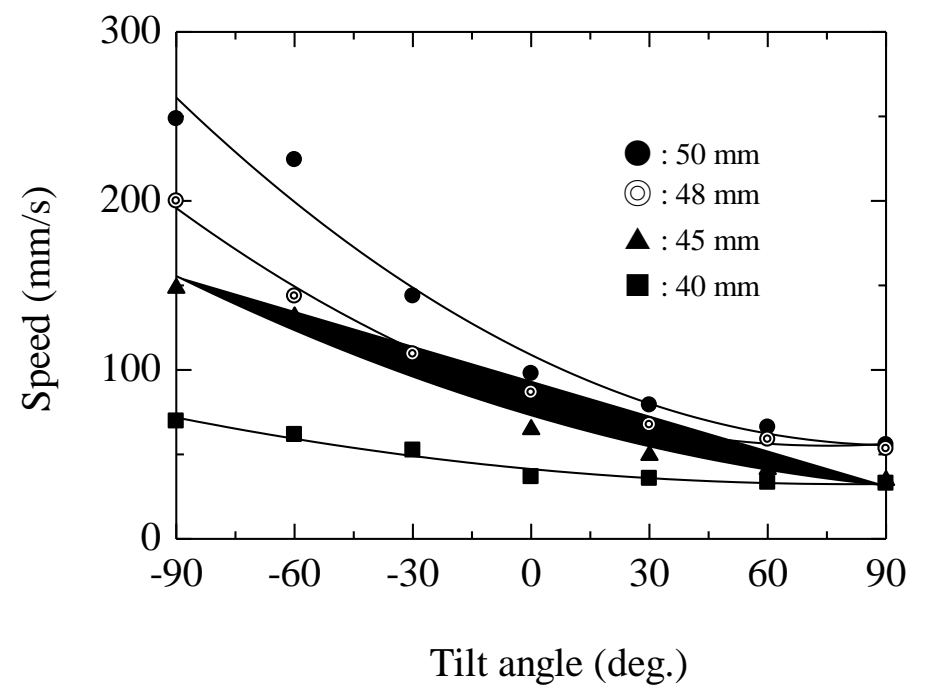

Figure 5. Relationship between tilt angle $\alpha$ and speed.

\section{MOVING PROPERTIES IN PIPE}

Finally, we operated the actuator system with the two CCD cameras and four LED lights. Figure 6 shows the concept of the electrical cable connection. The twenty electrical cables (six cables to the three electromagnets, four cables to the two CCD cameras, two cables to the four LED lights and eight cables to the four SMA wires) are necessary to drive the actuator system. Figure 7(a) shows the actuator system capable of inspection in a pipe of total length $10 \mathrm{~m}$. This actuator system was able to inspect the wall in the complex pipe with a T-joint, as shown in a previous study [15]. A pipe with one elbow curve was used in this paper, so that the effect of the electrical cable on the moving properties of the magnetic actuator system can be discussed. The pipe system was made by combining a vinyl chloride elbow pipe and a straight acrylic pipe. Figure 7(b) shows the image at half position A of pipe length $10 \mathrm{~m}$. Table 2 shows the relationship between the length of the electrical cable and the input voltage into electromagnet 1 of vibration component 1 . As the electrical cable becomes longer, the input voltage increases linearly.

On the other hand, Table 3 shows the relationship between the length of the electrical cable and the average speed of the actuator system in the horizontal direction. An electrical cable of diameter $0.12 \mathrm{~mm}$ was used in this paper. The total mass is $2 \mathrm{~g}$ when there are twenty cables because the mass per unit length of one electrical cable is $0.1 \mathrm{~g}$. Based on the result in Figure 5, this actuator system can move over $25 \mathrm{~m}$ in the 45 $\mathrm{mm}$ pipe. However, the moving properties considerably decrease even if the pipe system has only one elbow curve. The moving speed at the entrance of the pipe is fast; 
however, as the mileage of the actuator increases, the moving speed of the actuator system gradually decreases due to the influence of frictional force of the electrical cable. Furthermore, there is the problem of heat in the electromagnet.

Table 2. Relationship between length of electrical cable and input voltage into electromagnet 1 .

\begin{tabular}{ccc}
\hline Length $(\mathrm{m})$ & Voltage $(\mathrm{V})$ & Current $(\mathrm{A})$ \\
\hline 0.1 & 7.09 & 0.22 \\
1 & 7.78 & 0.22 \\
2 & 8.75 & 0.22 \\
4 & 9.49 & 0.22 \\
6 & 10.38 & 0.22 \\
8 & 11.32 & 0.22 \\
10 & 12.26 & 0.22 \\
\hline
\end{tabular}

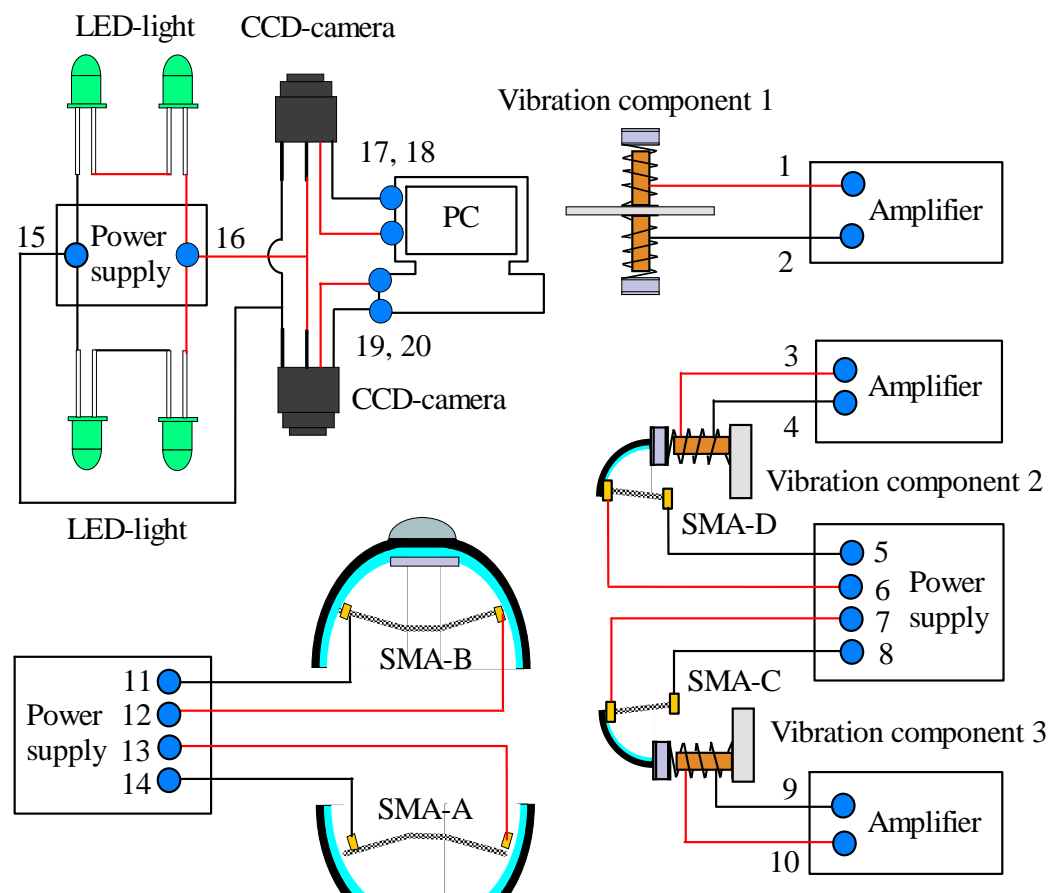

Figure 6. Concept of the electrical cable connection.

Figure 8 shows the relationship between the length of the electrical cable and the average speed in the horizontal direction when the input current into the electromagnet 1 was $0.22 \mathrm{~A}$. The measurement was carried out continually. The maximum traction length of cable for this actuator system was approximately $10 \mathrm{~m}$. Against the results mentioned above, the actuator system was not able to move a distance of $25 \mathrm{~m}$. We think that the electromagnetic force decreases due to the heat of the electromagnet. Measures to decrease the heat of the electromagnet are necessary 
when the actuator system moves over a long period. In future, an input current of less than $0.2 \mathrm{~A}$ is required.

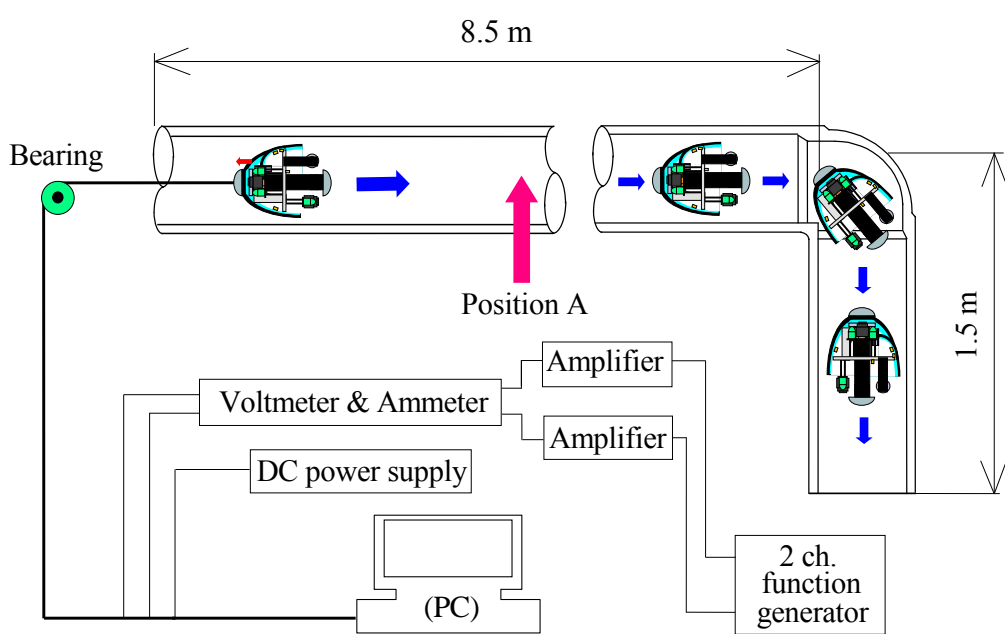

(a)

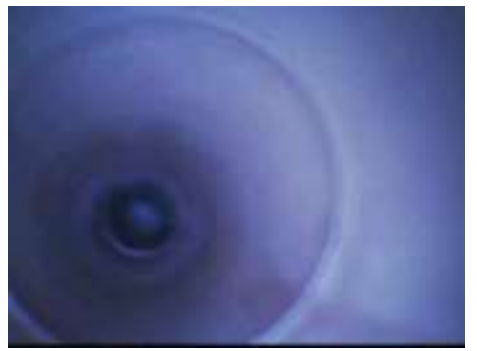

(b)

Figure 7. Experimental set-up: (a) actuator system; (b) photograph at position A.

Table 3. Relationship between horizontal speed and input voltage into electromagnet 1.

\begin{tabular}{cccc}
\hline Length $(\mathrm{m})$ & $\begin{array}{c}\text { Total mass of } \\
\text { twenty cables }(\mathrm{g})\end{array}$ & $\begin{array}{c}\text { Speed }(\mathrm{mm} / \mathrm{s}) \\
50 \mathrm{~mm}\end{array}$ & $\begin{array}{c}\text { Speed }(\mathrm{mm} / \mathrm{s}) \\
40 \mathrm{~mm}\end{array}$ \\
\hline 1 & 2 & 97.7 & 36.4 \\
2 & 4 & 77.9 & 33.9 \\
4 & 8 & 52.3 & 22.7 \\
6 & 12 & 35.1 & 15.6 \\
8 & 16 & 18.1 & 9.3 \\
10 & 20 & - & 4.8 \\
\hline
\end{tabular}

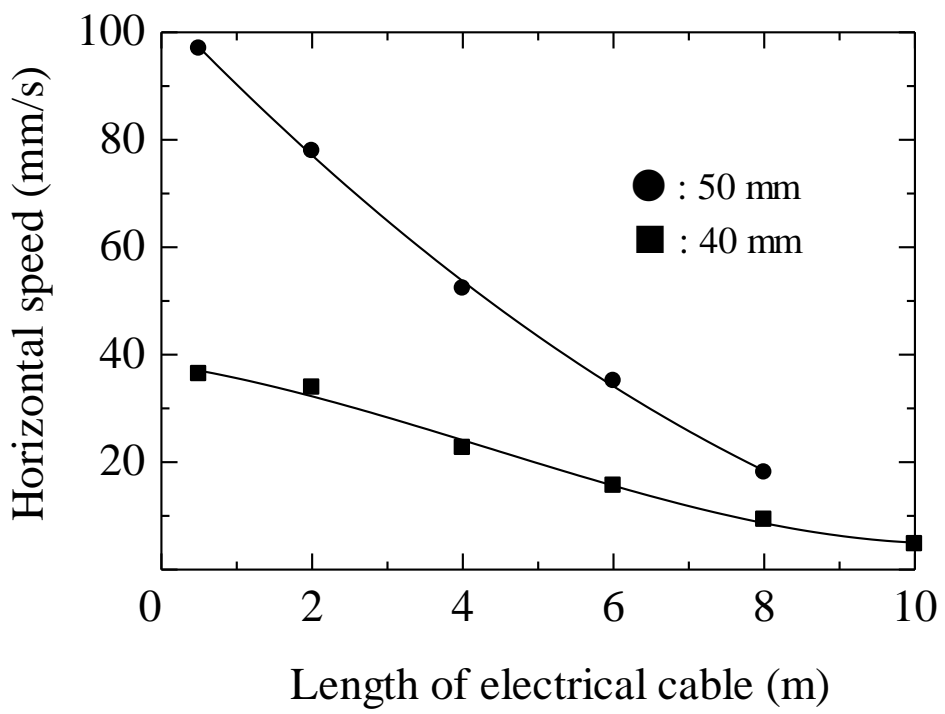

Figure 8. Relationship between length of electrical cable and horizontal speed. 


\section{CONCLUSIONS}

A magnetic actuator system has been proposed with a new type capable of inspection of a complex pipe. The effect of the electrical cable on the moving properties of the magnetic actuator was discussed experimentally. The experimental result shows that the magnetic actuator was able to climb at $15.3 \mathrm{~mm} / \mathrm{s}$ when pulling a load mass of $50 \mathrm{~g}$ with an inner pipe diameter of $40 \mathrm{~mm}$. The result demonstrated that the magnetic actuator system was able to inspect the complex pipe over a distance of $10 \mathrm{~m}$. In addition, we confirmed that the actuator system was able to move stably in the straight pipe. However, this actuator system is not able to inspect over long distances of approximately $10 \mathrm{~m}$. This is due to the influence of heat on the electromagnet.

\section{ACKNOWLEGEMENT}

The author would like to thanks to Tohoku Gakuin University, Japan for laboratory facilities and financial support.

\section{REFERENCES}

[1] Saito H, Sato K, Kudo K, Sato K. Fundamental study of mover travel inside a small diameter pipe. Transactions of the Japan Society of Mechanical Engineers. 2000;66:346-53.

[2] Fujita J, Shiraogawa Y, Yamamoto S, Kato T. Study of mobile mechanism with elastic fibers by using vibration. Transactions of the Japan Society of Mechanical Engineers. 2004;70:22-6.

[3] Jamil N, Yusoff AR, Mansor MH. Literature review of electromagnetic actuator force generation for dynamic modal testing applications. Journal of Mechanical Engineering and Sciences. 2012;3:311-9.

[4] Reynaerts D, Peirs J, Van Brussel H. Design of a shape memory actuated gastrointestinal intervention system. Eurosensors X. 1996:1181-4.

[5] Kwon J, Park S, Kim B, Park J-O. Bio-material property measurement system for locomotive mechanism in gastro-intestinal tract. IEEE International Conference on Robotics and Automation. 2005, p. 1315-20.

[6] Suzumori K, Wakimoto S, Tanaka M. In pipe inspection micro robot adaptable to changes in pipe diameter. IEEE International Conference on Robotics and Automation. 2003, p. 2735-40.

[7] Guo S, Pan Q. A paddling type of microrobot in pipe. International Conference on Intelligent Robots and Systems. 2005, p. 2265-70.

[8] Brunete I, Torres, J., Hernando, M. \& Gambao, E. A 2 DOF servomotor-based module for pipe inspection modular micro-robots. International Conference on Intelligent Robots and Systems. 2006.

[9] Choi H, Roh S. In-pipe robot with active steering capability for moving inside of pipelines. Bioinspiration and Robotics Walking and Climbing Robots. 2007;23:375-400.

[10] Miyagawa T, Iwatsuki N. Moving characteristics in bent pipes of in-pipe mobile robot with wheel drive mechanism using planetary gear drive. Journal of the Japan Society for Precision Engineering. 2008;74:1346-50.

[11] Bocko J, Kelemen M, Kelemenova T, Jezný J. Wheeled locomotion inside pipe. Bulletin of Applied Mechanics. 2009;5:34-6. 
[12] Yaguchi H, Sato N. Globular magnetic actuator capable of free movement in a complex pipe. IEEE Transactions on Magnetics. 2010;46:1350-5.

[13] Yaguchi H, Sato N, Shikoda A. Magnetic actuator group of globular type capable of free movement in a complex pipe. IEEE Transactions on Magnetics. 2011;47:4159-62.

[14] Yaguchi H, Kamata K. In-piping magnetic actuator capable of inspection in a thin complex pipe. Mechanical Engineering Research. 2012;2:1-9.

[15] Yaguchi H, Sasaki K. New type of magnetic actuator system for inspection in a complex pipe. IEEE Transactions on Magnetics. 2013;49:3905-8. 